\title{
Estratégias utilizadas por enfermeiras para minimizar a ocorrência de delirium em pacientes críticos
}

Strategies used by nurses to minimize the occurrence of delirium in critically ill patients

Estrategias utilizadas por las enfermeras para minimizar la aparición de delirio en pacientes críticos

\section{Katherine Pithon Oliveira ${ }^{1}$, Carina Marinho Picanço ${ }^{2}$, Adriana Ribeiro Oliveira ${ }^{3}$, Ylara Idalina Silva de Assis ${ }^{4}$, Ana Cláudia Fonseca de Souza ${ }^{5}$, Aldacy Gonçalves Ribeiro ${ }^{6}$}

Resumo: Objetivo: descrever as estratégias utilizadas por enfermeiras para minimizar a ocorrência de delirium em pacientes internados em unidade de terapia intensiva (UTI). Método: estudo exploratório-descritivo, de abordagem qualitativa, desenvolvido em um hospital de ensino, público, de grande porte da cidade de Salvador. Foi realizado nos meses de setembro e outubro de 2018, participaram do estudo 16 enfermeiras. Os dados foram coletados mediante entrevista semiestruturada e analisados por meio da "Técnica de Análise de Conteúdo Temática". Resultados: da análise dos dados emergiram duas categorias temáticas, denominadas: "Desconhecimento sobre monitorização do delirium em UTI" e "Estratégias das enfermeiras para minimizar a ocorrência de delirium em UTI". Conclusão: apesar do pouco conhecimento das enfermeiras sobre o delirium existe uma coerência quantos aos métodos de intervenções para preveni-lo. A implementação de protocolos e atividades educativas são imprescindíveis para empoderar o enfermeiro quanto às intervenções realizadas.

Descritores: Delirium; Unidade de Terapia Intensiva; Cuidados de Enfermagem; Enfermagem de cuidados críticos; Pesquisa Qualitativa

\footnotetext{
${ }^{1}$ Enfermeira, especialista em terapia intensiva. Hospital Geral Roberto Santos, Salvador, Bahia, Brasil. E-mail: kathypithon@hotmail.com. https://orcid.org/0000-0002-2392-5263

2 Enfermeira, mestra em enfermagem. Hospital Geral Roberto Santos, Salvador, Bahia, Brasil. E-mail: carinampicanco@gmail.com. https://orcid.org/0000-0003-4758-4333

3 Enfermeira, especialista em terapia intensiva. Hospital Geral Roberto Santos, Salvador, Bahia, Brasil. E-mail: enfa.oliveira@gmail.com. https://orcid.org/0000-0002-9685-2085

${ }^{4}$ Enfermeira, especialista em enfermagem do trabalho. Hospital Geral Roberto Santos, Salvador, Bahia, Brasil. E-mail: ylara.i.assis@gmail.com. https://orcid.org/0000-0003-4800-7095

${ }^{5}$ Enfermeira, especialista em terapia intensiva. Hospital Geral Roberto Santos, Salvador, Bahia, Brasil. E-mail: caufs@yahoo.com.br. https://orcid.org/0000-0002-9435-6133

6 Enfermeira, mestra em enfermagem. Hospital Geral Roberto Santos, Salvador, Bahia, Brasil. E-mail: aldacygoncalves@hotmail.com. https://orcid.org/0000-0002-7021-6099
} 


\begin{abstract}
Objective: to describe the strategies used by nurses to minimize the occurrence of delirium in patients admitted to the intensive care unit (ICU). Method: exploratory-descriptive study, with a qualitative approach, developed in a large public teaching hospital in the city of Salvador. It was carried out in September and October 2018, 16 nurses participated in the study. Data were collected through semi-structured interviews and analyzed using the "Thematic Content Analysis Technique". Results: from the data analysis, two thematic categories emerged, named: "Lack of knowledge about monitoring delirium in the ICU" and "Strategies of nurses to minimize the occurrence of delirium in the ICU”. Conclusion: although the nurses' lack of knowledge about delirium, there is a coherence in the methods of interventions to prevent it. The implementation of protocols and educational activities are essential to empower nurses regarding the interventions performed.

Descriptors: Delirium; Intensive Care Units; Cuidados de Enfermagem; Nursing Care; Critical Care Nursing; Qualitative Research
\end{abstract}

Resumen: Objetivo: describir estrategias utilizadas por enfermeras para minimizar la aparición de delirio en pacientes ingresados en unidad de cuidados intensivos (UCI). Método: estudio exploratorio, descriptivo, con enfoque cualitativo, desarrollado en un gran hospital público docente de la ciudad de Salvador. Se realizó en septiembre y octubre de 2018, con 16 enfermeras participantes del estudio. Los datos fueron recolectados por entrevistas semiestructuradas y analizados utilizando la "Técnica de análisis de contenido temático". Resultados: del análisis de datos, surgieron dos categorías temáticas, llamadas: "Falta de conocimiento sobre el monitoreo del delirio en la UCI" y "Estrategias de las enfermeras para minimizar la aparición de delirio en la UCI". Conclusión: a pesar de la falta de conocimiento de las enfermeras sobre el delirio, existe coherencia en los métodos de intervención para prevenirlo. La implementación de protocolos y actividades educativas es esencial para empoderar a las enfermeras con respecto a las intervenciones realizadas.

Descriptores: Delirio; Unidades de Cuidados Intensivos; Atención de Enfermería; Enfermería de Cuidados Críticos; Investigación Cualitativa

\title{
Introdução
}

O delirium é uma disfunção neurológica aguda mais frequentemente observada em pacientes internados em Unidades de Terapia Intensiva (UTI), caracterizado por alterações transitórias da consciência e cognição, flutuação do estado mental, desatenção e pensamento desorganizado, em geral, por curto período de tempo e tende a flutuar ao longo do dia. ${ }^{1}$ Estudo publicado demonstra que o delirium nas UTI desencadeia importante declínio funcional e está associado à maior período de ventilação mecânica, hospitalização prolongada, aumento dos custos e maior mortalidade. ${ }^{2}$

Achados apontam que a incidência do delirium em pacientes críticos varia entre $47 \% \mathrm{e}$ $80 \%$ e sua prevalência entre $20 \%$ e $80 \%{ }^{3}$ Entretanto, permanece subdiagnosticado um percentual 
3 | Oliveira KP, Picanço CM, Oliveira AR, Assis YIS, Souza ACF, Ribeiro AG

compreendido entre $25 \%$ e $75 \%$. Este dado pode estar relacionado devido a flutuação do próprio quadro de delirium, associado ao conhecimento insuficiente por parte dos profissionais de saúde sobre a patologia, bem como a baixa adesão às ferramentas diagnósticas. ${ }^{4}$

De acordo com o Diagnostic and Statistical Manual of Mental Disorders - 5 (DSM-5) é definido como: 1)Alteração da consciência com diminuição da capacidade de manter, alterar ou focar a atenção; 2) Alteração da cognição (memória, orientação, linguagem) ou 3) desenvolvimento de alteração da percepção que não pode ser explicada por quadro demencial estabelecido, pré-existente ou em evolução. As alterações descritas desenvolvem-se num curto período de tempo, geralmente horas a dias, e tendem a flutuar ao longo do dia. É considerado também, como uma manifestação heterogênea e, para melhor caracterizá-lo foram descritos três subtipos: hiperativo (agitação), hipoativo (apatia) e misto (com características das duas formas).

Os fatores de risco para delirium se apresentam em diferentes contextos clínicos e sua causa é multifatorial, entretanto, alguns tipos de pacientes, devido às características particulares, intrínsecas e extrínsecas, apresentam uma maior incidência para o desenvolvimento. No entanto, o seu desenvolvimento depende de uma interação entre a vulnerabilidade do paciente (fatores predisponentes) e os insultos da doença aguda (fatores precipitantes), de maneira que o paciente menos vulnerável precisa de insultos mais intensos e os pacientes mais vulneráveis podem apresentar delirium com insultos menores. ${ }^{6}$

A prevenção do delirium está diretamente ligada ao reconhecimento dos fatores de risco modificáveis, àqueles que são passíveis de intervenções, em que o tratamento mais efetivo ainda é o preventivo. As estratégias de tratamento podem ser farmacológicas e não-farmacológicos. Dentre as farmacológicas destacam-se o uso do haloperidol, por ter um custo menor, porém, o sedativo de escolha é a dexmetomidina. Já, os benzodiazepínicos devem ser evitados devido ao efeito colateral de confusão mental. As estratégias não-farmacológicas se dão por meio do atendimento psicológico na UTI, visita prolongada do familiar, promoção de ambiente calmo, 
Estratégias utilizadas por enfermeiras para minimizar a ocorrência de delirium em... | 4

silencioso e com redução de estímulos sonoros e luminosos. Além de outras ações como evitar a utilização da contenção física, incentivo à mobilização precoce, implementação da musicoterapia, utilização precoce de métodos de avaliação como o Confusion Assessment Method in a Intensive Care Unit (CAM-ICU), uso de óculos e aparelhos auditivos, remoção precoce de dispositivos invasivos e correção dos distúrbios hidroeletrolíticos. ${ }^{7-8}$

Baseado no programa chamado "Liberation da UTI", a Society of Care Medicine (SCCM) atualizou as "Diretrizes de Prática Clínica para Manejo da Dor, Agitação e Delirium", em que descrevem as melhores evidências disponíveis para abordar os elementos imprescindíveis para assegurar o conforto e a segurança dos pacientes internados em terapia intensiva. Nessas diretrizes foram acrescentados dois tópicos de atenção clínica relacionados à reabilitação/mobilização e sono, publicada como: "Diretrizes de Prática Clínica para a Prevenção e Tratamento da Dor, Agitação/Sedação, Delirium, Imobilidade e Interrupção do Sono em Pacientes Adultos na UTI (PADIS). ${ }^{10}$

$\mathrm{Na}$ UTI as enfermeiras, geralmente são as primeiras a identificar as alterações comportamentais apresentadas pelos pacientes, preocupando-se com quadros confusionais ou de agitação em razão de eventos adversos que podem colocar em risco sua segurança e ocasionar consequências deletérias. ${ }^{11}$ Assim sendo, evidencia-se a importância e a necessidade da identificação precoce do delirium realizada pelas enfermeiras por meio de instrumentos válidos, bem como o conhecimento dos possíveis fatores relacionados à ocorrência do delirium em pacientes internados nas unidades de cuidados intensivos.

Ademais, durante a vivência enquanto residente em enfermagem em terapia intensiva, foi possível perceber uma lacuna no que tange às ações e estratégias de intervenção e avaliação sistemática para diagnóstico precoce de delirium, bem como inexistência de protocolos para a sua prevenção. Desse modo, este estudo parte da seguinte questão de pesquisa: Quais as estratégias utilizadas pelas enfermeiras para minimizar a ocorrência de delirium em pacientes 
5 | Oliveira KP, Picanço CM, Oliveira AR, Assis YIS, Souza ACF, Ribeiro AG

internados em unidade de terapia intensiva? Para tanto, objetiva descrever as estratégias utilizadas por enfermeiras para minimizar a ocorrência de delirium em pacientes internados em unidade de terapia intensiva.

\section{Método}

Trata-se de um estudo qualitativo, do tipo exploratório-descritivo, realizado em uma UTI adulto de um hospital público da cidade de Salvador, Bahia, Brasil. Essa Instituição foi inaugurada em 1979, e atualmente possui 630 leitos disponíveis para atendimento de referência em urgência/emergência clínica, obstétrica e traumática, além das especialidades de neurologia, infectologia, obstetrícia, nefrologia, gastro-hepática, hemorragias digestivas e centro de intoxicação exógena. As Unidades de Terapia Intensiva correspondem ao maior complexo de UTI do Estado da Bahia, com 109 leitos, divididos entre UTI Adulto (geral, cirúrgica, cardiovascular e neurológica), pediátrica e neonatal.

A pesquisa seguiu as normas éticas preconizadas, atendendo as conformidades dispostas no Conselho Nacional de Saúde (CNS) na Resolução $n^{\circ} 466 / 12$. O projeto foi aprovado pelo Comitê de ética e Pesquisa do referido hospital sob o número do parecer 2.824.588.

Estabeleceu-se, como critério de inclusão, ser enfermeira assistencial de uma UTI adulto. Excluíram-se todas as enfermeiras que não atuavam diretamente com o cuidado ao paciente crítico e/ou estavam afastadas por férias, licença prêmio e licença médica. O quantitativo total de possíveis participantes eram 22, porém de acordo com o critério de saturação dos dados, participaram do estudo 16 , visto que as respostas começaram a se repetir.

A coleta dos dados ocorreu nos meses de setembro e outubro de 2018, por meio de uma entrevista semiestruturada, a partir de questões sobre as estratégias utilizadas pelas enfermeiras para minimizar o risco de delirium na UTI, como: 1. Você conhece algum instrumento para monitoração de delirium na UTI? 2. No seu processo de trabalho, na UTI, você inclui a 
Estratégias utilizadas por enfermeiras para minimizar a ocorrência de delirium em... $\mid 6$

monitoração de delirium? 3. Durante sua assistência de enfermagem ao paciente crítico existe alguma estratégia de trabalho que você utiliza para prevenir a ocorrência de delirium? Se não, por quê? Além de responderem um formulário com informações para caracterização sociodemográfica, referente à idade, gênero, unidade de alocação, tempo de graduação, especialização e tempo de serviço.

Os dados foram coletados pela pesquisadora responsável. As entrevistas foram realizadas em sala reservada, com datas e horários previamente agendados, conforme a disponibilidade dos participantes. Cada entrevista teve duração média de 30 minutos. As participantes foram incluídas no estudo após assinarem o termo de consentimento livre e esclarecido e receberam identificações fictícias, como codinomes de flores. Estas foram gravadas e, posteriormente às entrevistas, iniciou-se a transcrição das falas, com auxílio de um fone de ouvidos e de um telefone celular, por meio do aplicativo "Voz a Texto Texto a Voz PDF", a fim de garantir exatidão e minimizar possíveis erros, todo o material transcrito passou por dupla revisão.

O processo de análise dos dados se deu através da “Técnica de Análise de Conteúdo Temática” ${ }^{12}$ o qual organizou-se conforme as seguintes etapas: 1. Pré-análise: partiu do processo da transcrição das entrevistas e leitura flutuante das mesmas, quando o conteúdo das respostas pôde se tornar mais claro. 2. Exploração do material: em que foi possível identificar as unidades de registro (palavras) as quais foram codificadas de acordo com as unidades de contexto (por meio de recortes das falas das entrevistas). 3. Tratamento dos resultados: nesta etapa ocorreu a condensação e o destaque das informações para análise, resultando nas interpretações inferenciais; o momento da intuição, da análise reflexiva e crítica, o que permitiu a categorização das ideias.

Por se tratar de uma pesquisa de campo, com análise de dados coletados por meio do conhecimento dos participantes, existe risco de exposição desses dados, portanto, foi assegurado as participantes o sigilo de suas informações e, garantido que, após a transcrição e a 
7 | Oliveira KP, Picanço CM, Oliveira AR, Assis YIS, Souza ACF, Ribeiro AG

conferência da exatidão do processo de transferência das falas para a escrita, o áudio seria descartado imediatamente, sendo mantido arquivado por cinco anos apenas o registro impresso.

\section{Resultados e discussões}

\section{Caracterização dos participantes}

As participantes da pesquisa eram todas do sexo feminino e apresentaram idade média de 34 anos. Quanto ao tempo de formação possuíam em média 5 anos de profissão. O tempo de atuação dessas profissionais em terapia intensiva foi bastante heterogêneo, cinco participantes tinham menos de um ano de atuação profissional, dez entre um e quatro anos de atuação e, apenas uma referiu ter oito anos de experiência. Já, referente à carga horária, a maioria (12) cumprem 30 horas semanais e, quatro delas possuíam mais de um vínculo, contabilizando carga horária total de 74 horas semanais.

É importante ressaltar que a maioria (treze) das participantes possuem título de especialista em terapia intensiva. Portanto, esperou-se que a maior parte das profissionais soubesse reconhecer os sinais e sintomas do delirium, seus fatores de risco, assim como os cuidados de enfermagem para prevenção deste distúrbio.

A partir da análise das entrevistas emergiram duas categorias temáticas, denominadas: Desconhecimento sobre monitorização do delirium em UTI e Estratégias das enfermeiras para minimizar a ocorrência de delirium em UTI; conforme apresentação a seguir.

\section{Desconhecimento sobre monitorização do delirium em UTI}

Ao analisar as respostas das enfermeiras sobre conhecer algum instrumento para monitoramento de delirium na UTI e sua utilização, as entrevistadas, em sua maioria, não tinham conhecimento sobre a existência de um instrumento para monitorização do delirium e sua utilização na prática assistencial em pacientes internados em UTI. 
Estratégias utilizadas por enfermeiras para minimizar a ocorrência de delirium em... $\mid 8$

Não, até hoje não tem nenhum instrumento que eu conheço não. (Girassol)

Instrumento específico, não. Imagino que exista estratégia? Mas na aplicabilidade assim, na prática, pelo menos pela enfermagem, não conheço não. (Rosa)

Equipamento mesmo, equipamento? Só o profissional mesmo é que percebe quando na conversa com o paciente, no discurso dele, é que percebe que ele está tendo um episódio de delirium. (Lírio)

A enfermagem tem um papel indispensável na avaliação e identificação do delirium, visto ser uma equipe profissional que permanece 24 horas com o paciente, qualquer oscilação do estado de humor que o mesmo apresente, pode ser facilmente identificável. ${ }^{13}$ A maioria das enfermeiras sabe reconhecer alguns sinais de delirium, porém desconhecem o delirium como uma desordem orgânica, além disso, desconhecem métodos de avaliação, impondo apenas ao médico o diagnóstico, além de desconhecerem métodos de prevenção e cuidados do paciente com delirium. ${ }^{14}$

Para facilitar o diagnóstico do delirium em pacientes admitidos na UTI, foi criado um instrumento de observação e avaliação de pacientes graves intubados sob ventilação mecânica, baseado nas principais características do Diagnostic and Statistical Manual of Menthal Disorders, Fouth Edition (DSM-IV), o CAM-ICU. Essa ferramenta foi validada para língua portuguesa em 2011, os resultados demonstraram alta especificidade (96,2\%), boa sensibilidade (72,5\%), além de bom poder preditivo quando a escala é utilizada de forma sistemática ${ }^{6}$. O instrumento CAM-ICU favorece uma comunicação por meio de uma linguagem uniforme na equipe multidisciplinar, sendo considerado um instrumento "padrão-ouro" do reconhecimento da síndrome em pacientes graves. ${ }^{15}$ 
9 | Oliveira KP, Picanço CM, Oliveira AR, Assis YIS, Souza ACF, Ribeiro AG

Quanto a utilização de algum instrumento para sua avaliação ou monitorização de delirium, as entrevistadas referiram não utilizar o CAM-ICU ou qualquer outro método para seu diagnóstico. Vale ressaltar que esta prática não é implementada no cenário deste estudo, como demonstrado nas falas a seguir:

Conheço uma escala que monitoriza o delirium, porém, a gente não utiliza na UTI que eu trabalho, porque não é protocolo. (Azaleia)

Impresso específico não tem. A gente consegue identificar através da condição clínica do paciente, mas impresso específico, não. (Rosa)

Não, não existe nenhum protocolo. (Onze-horas)

Corroborando as evidências da presente pesquisa, em relação ao conhecimento e uso do CAM - ICU, um estudo realizado no hospital geral do Estado de Santa Catarina evidenciou o conhecimento insuficiente sobre a escala diagnóstica e a importância da avaliação do delirium a beira do leito. Autores discutem que para prevenção e controle do delirium é necessário não apenas o conhecimento científico, mas também mudanças estruturais e comportamentais dos profissionais. O subdiagnóstico está ligado à falta de conhecimento, alinhado às condutas equivocadas, como o uso excessivo de sedação, contenção mecânica mal executada, entre outros aspectos relacionados à má qualidade da assistência. ${ }^{16}$

Um estudo realizado com enfermeiras sobre a avaliação do delirium na UTI, concluiu que a prática de identificação por meio do método já supracitado foi efetiva na redução do uso de sedativos, de contenção mecânica e no melhor manejo de aspectos relacionados ao ambiente, por influência das intervenções prescritas pela enfermeira. ${ }^{17}$ 
Assim, a enfermeira, como integrante da equipe multiprofissional, tem o foco do seu trabalho no atendimento assistencial por meio do cuidado e dos julgamentos clínicos. Além de possuir o papel de diagnosticador de problemas relacionados ao paciente. Para isso, faz uso do pensamento crítico a fim de determinar os diagnósticos mais acurados, promover orientações e intervenções de enfermagem com vistas a alcançar os melhores resultados para o paciente e a família. ${ }^{18}$

\section{Estratégias das enfermeiras para minimizar a ocorrência de delirium em UTI}

Os depoimentos nessa categoria permitiram apreender a percepção que os profissionais em estudo têm sobre o delirium, bem como as experiências e as estratégias utilizadas na prevenção desta injúria. Quando questionadas sobre alguma estratégia de trabalho que utiliza para a prevenção e ocorrência de delirium, as respostas versaram nos eixos família mobilização - ambiente. Sobre este primeiro aspecto, família, as participantes do estudo enfatizaram sobre a importância da presença da família junto ao paciente crítico, foi destacado que a presença desta contribui para que o paciente se mantenha orientado no tempo e espaço.

[...] dá noção de tempo, de espaço para ele, sempre falando onde que ele está, o dia que é, as horas, e tenta sempre aproximar a família nesse meio para que ele não entre em delirium. Eu acho que toda unidade de terapia intensiva deve ter essa visita ampliada para os pacientes, com o acompanhamento da psicologia porque a família é muito importante nesse processo, vamos se dizer assim. (Girassol) 
11 | Oliveira KP, Picanço CM, Oliveira AR, Assis YIS, Souza ACF, Ribeiro AG

[...] atualizar ele na questão do tempo, onde está, conversar sobre família? Manter o diálogo e a assistência humanizada [...]. (Copode-leite)

Então assim, é [...] está mais próximo do paciente, ter mais esse acolhimento, as visitas poderiam ser um pouco mais estendidas [...]. (Lírio)

[...] permitir que visitas sejam realizadas mesmo fora do horário [...] a gente já sabe que esta questão de visita ajuda muito na recuperação do paciente, em vez de atrapalhar, como a gente antes imaginava [...]. (Cravo)

O conhecimento das enfermeiras sobre os fatores de risco que podem favorecer a ocorrência do delirium é imprescindível para que realizem uma sistematização da assistência de enfermagem (SAE) adequada aos pacientes, bem como uma atenção especial quanto à necessidade de maior suporte e presença de seus familiares. ${ }^{19}$ A promoção da visita de familiares é ratificada em um estudo ${ }^{20}$ que também considerou como um fator de risco modificável pelas enfermeiras e, foi observado que existe uma percentagem significativa (44\%) de pacientes que não recebiam visitas e presença de delirium.

Ao redor do mundo, experiências de flexibilização dos horários de visitação familiar em UTI têm- se mostrado seguras e reprodutíveis. Congruente com esse cenário, o projeto "UTI Visitas" foi elaborado com o objetivo de aumentar o tempo de visita familiar em UTI brasileiras, a fim de tornar o atendimento humanizado e centrado nas necessidades do paciente e sua família. ${ }^{21}$ Está participando deste projeto a UTI cirúrgica do hospital, lócus desta pesquisa, que desde 2017, vem apresentando a proposta de visita ampliada, e tem como meta a qualificação do cuidado ao paciente crítico. 
Estratégias utilizadas por enfermeiras para minimizar a ocorrência de delirium em... $\mid 12$

O segundo aspecto referente às estratégias das enfermeiras para minimizar o risco de delirium, está relacionado à contenção mecânica, em que as entrevistadas referem evitar essa prática, mantendo-a apenas em caso de risco de exteriorização de dispositivos.

$$
\begin{aligned}
& \text { [...] evito deixar os pacientes contidos sem necessidade? O } \\
& \text { paciente que eu percebo que não está agitado, que tem nível de } \\
& \text { consciência para poder se proteger e que não vai, não tem risco de } \\
& \text { exteriorizar dispositivos, de se extubar acidentalmente, eu busco } \\
& \text { avaliar isso bem criticamente junto à equipe multi, para que o } \\
& \text { paciente não fique contido? (Orquídea) } \\
& \text { [...] não deixar o paciente contido desnecessariamente [...] não } \\
& \text { conter ele mecanicamente de forma desnecessária [...] (Cravo) }
\end{aligned}
$$

$\mathrm{Na}$ prática clínica, a agitação psicomotora deve ser considerada e, normalmente, necessita de manejo farmacológico devido ao risco de ocorrência de eventos adversos, tais como exteriorização de dispositivos e até mesmo a queda do paciente. No entanto, a equipe multiprofissional necessita realizar ações que possibilite um ambiente tranquilo, para que o paciente permaneça calmo, desperto e colaborativo para desenvolver todas as intervenções pertinentes para prevenção e tratamento de delirium, tais como mobilização precoce, interação com a família e reorientação. ${ }^{22}$

Dessa forma, observa-se o uso da contenção física rotineiramente e indiscriminadamente nos pacientes internados na UTI, mesmo sabendo que é um dos principais fatores precipitantes para ocorrência do delirium. O uso de restrições é um tema complexo, que vai além do cuidado de enfermagem, abrangendo questões físicas, psicológicas, legais e éticas.

Assim, o Conselho Federal de Enfermagem (COFEN), por meio da resolução do no 427 de 2012, resolve que os profissionais da enfermagem somente podem empregar a contenção mecânica 
sob supervisão direta do enfermeiro e em conformidade com os protocolos estabelecidos pelas instituições de saúde, salvo casos de urgência ou emergência. Recomenda ainda que a contenção mecânica deve ser utilizada apenas quando for o único meio possível para prevenção de danos aos pacientes ou aos demais, não devendo ser prolongada, tampouco utilizada com o propósito de disciplinar, punir ou coagir ou mesmo por conveniência da equipe. ${ }^{23}$

Em um estudo ${ }^{16}$ com enfermeiras, observou-se que existe a preocupação pelo uso inadequado das restrições mecânicas e, ao mesmo tempo, o temor pela responsabilização da enfermagem caso algo ocorra ao paciente não devidamente restrito. Para tanto, é importante ressaltar que a restrição física pode ser necessária em diversas situações para evitar a ocorrência de eventos adversos, como: queda, extubação acidental, retirada de dispositivos, dentre outros. No entanto, a equipe de enfermagem juntamente com a equipe multidisciplinar deve avaliar criteriosamente cada caso antes de optarem por esse tipo de restrição.

Em relação ao ambiente e higiene do sono, destaca-se as intervenções que podem ser implementadas, como proporcionar um meio interativo de comunicação, a exemplo da televisão, reduzir e controlar ruídos, proporcionar um ambiente com visibilidade da luz solar, reduzir a luminosidade no período noturno, como descrito nas falas a seguir:

[...] eu acho que todo esse ambiente fechado favorece para que aumente esses episódios de delirium no paciente, ter mais janelas onde ele possa ver a luz do dia [...] (Lírio)

[...], mas eu acho que se tivesse um meio de comunicação, uma televisão, algo do tipo que paciente pudesse ter uma noção ambiente fechado tipo uma UTI, eu acho que é muito mais fácil ir o delirium por conta do ambiente e tudo [...] confinamento. (Rosa Amarela) 
Estratégias utilizadas por enfermeiras para minimizar a ocorrência de delirium em... $\mid 14$

[...] às vezes há luz demais, o barulho do monitor, eu sempre procuro deixar o monitor com os alarmes de acordo, para não está o tempo inteiro ali [...] a luz, quase $100 \%$ dos pacientes que estão, quase todos a gente coloca fronha, fronha não, travessa por causa da luz incomodando [...] realmente muda o sono, a qualidade do sono. (Violeta)

Os cuidados de enfermagem relativos ao ambiente hospitalar podem ser um fator preventivo para o desenvolvimento do delirium. Deste modo, o cuidado com o ambiente é indicado para minimizar os fatores que podem acentuar o quadro. Ao criar e manter um ambiente mais familiar, permitir que a família exponha fotos de família ou objetos preferidos, são atitudes simples, mas que tem impacto positivo em relação a diminuição do risco de os pacientes apresentarem desorientação e confusão mental.

Outrossim, os pacientes criticamente enfermos apresentam uma alta probabilidade de apresentar delirium devido à dinâmica da unidade, por se tratar de um ambiente ruidoso e de grande agitação, o que acaba provocando no paciente um sono inconstante, além de favorecer o surgimento dos fatores precipitantes desse agravo. Sendo assim é necessário controlar esse ambiente no tangente aos ruídos e outros fatores que dificultem o padrão de sono e repouso dos doentes. ${ }^{6}$

Nesse sentido, é imprescindível que a equipe de enfermagem tenha maior atenção para que o ambiente, principalmente durante a noite, seja calmo e as luzes amenizadas, assim como adequar os horários de medicações e procedimentos, no intuito de proporcionar um sono eficaz, pois sabe-se que a privação do sono provoca desatenção e disfunção cognitiva, que são características que podem estar presentes nos pacientes com delirium. 


\section{Conclusão}

Os resultados permitiram concluir que, embora exista uma lacuna no conhecimento das enfermeiras em diagnosticar o delirium, há uma coerência quanto às estratégias para preveni-lo, porém, as ações presentes nos discursos das participantes são superficiais. As enfermeiras destacam a presença da família, evitar o uso indiscriminado de contenção mecânica, controle do ambiente quanto a ruídos e luminosidade e higiene do sono, como medidas preventivas para a minimização de delirium em pacientes críticos.

Os dados deste estudo ratificam a necessidade e importância de educação permanente que envolva a temática delirium para a equipe de enfermagem, de modo que seja possibilitado a organização do processo de enfermagem ao que se refere a conhecimento e aplicação de estratégias que permitam assim a identificação precoce do delirium de modo que o paciente seja assistido de forma segura e livre de iatrogenia. Assim, será possível melhorar os desfechos clínicos dos pacientes críticos em delirium, mantendo assim uma assistência qualificada e segura.

Portanto, torna-se imprescindível que as instituições de saúde desenvolvam protocolos para que o score de avaliação dessa injúria seja realizado, juntamente com atividades educativas, pois por meio da instrumentalização do conhecimento sobre delirium, o processo de trabalho tornar-se-á mais objetivo e científico, não sendo verificado apenas por meio da observação empírica.

\section{Referências}

1. Mori S, Takeda JRT, Carrara FSA, Cohrs CR, Zanei SSV, Whitaker IY. Incidence and factors related to delirium in an intensive care unit. Rev Esc Enferm USP. [internet] 2016 [acesso em 2018 Ago 1];50(4): 585-591. Disponível em: http://www.scielo.br/pdf/reeusp/v50n4/pt_0080-6234-reeusp-50-04-0587.pdf DOI: http://dx.doi.org/10.1590/S0080-623420160000500014

2. Brummel NE, Vasilevskis EE, Han JH, Boehm L, Pun BT, Ely EW. Implementing delirium screening in the ICU. Crit Care Med. [internet] 2013 Set [acesso jun 2019 1];41(9):2196-208. Disponível em: https://www.ncbi.nlm.nih.gov/pubmed/23896832doi: 10.1097 / CCM.0b013e31829a6f1e. 
Estratégias utilizadas por enfermeiras para minimizar a ocorrência de delirium em... $\mid 16$

3. Faria RSB, Moreno RP. Delirium na unidade de cuidados intensivos: uma realidade subdiagnósticada. Rev. bras. ter. intensiva [internet]. 2013 [acesso em Abril 2018 8];25(2):137-147. ISSN 0103-507X. Disponível em: http://dx.doi.org/10.5935/0103-507X.20130025.

4. Sanchez JC, Gonzalez MI, Gutierrez JC. Delírium en pacientes mayores de 60 años en un hospital público de tercer nivel en la ciudad de Pereira (Colombia): subdiagnóstico y subregistro. rev.colomb. psiquiatr. [internet]. 2013 [acesso Jun 2018 15];42(2):191-197. ISSN 0034-7450. Disponível em: http://www.scielo.org.co/scielo.php?pid=S0034-74502013000200006\&script=sci_abstract\&tlng=pt

5. American Psychiatric Association (Washington). Diagnosticand Statistical Manual of Mental Disorders: (DSM V) Revised. 5. ed. 2013.

6. Flôres DG, Capone NA. Delirium no paciente grave. Clínicas de Medicina Intensiva Brasileira (CMIB). 2013, 23(18). Associação de Medicina Intensiva Brasileira (AMIB). São Paulo: Atheneu, 2013.

7. Pincelli EL, Waters C, Hupsel ZN. Ações de enfermagem na prevenção do delirium em pacientes na Unidade de Terapia Intensiva. Arq Med Hosp Fac Cienc Med Santa Casa São Paulo. [internet]. 2015 [acesso Out 2019]; 60:131-9. Disponível em: http://arquivosmedicos.fcmsantacasasp.edu.br.

8. Salluh JIF, Pandharipande P. Prevenção do delirium em pacientes críticos: um recomeço? Rev Bras Ter Intensiva. [internet] 2012 [acesso Nov 2018 23];24(1). Disponível em: http://dx.doi.org/10.1590/S0103507X2012000100001.

9. Barr J, Fraser G, Puntilho K. Clinical Practice Guidelines for the Management of Pain, Agitation, and Delirium in Adult Patients in the Intensive Care Unit. Critical Care Medicine. [internet] 2013 Jan [acesso Jun 2018 13];41(1) 263-306. Disponível em: https://www.ncbi.nlm.nih.gov/pubmed/23269131doi: 10.1097/CCM.0b013e3182783b72

10. Devlin JW, Skrobik Y, Gélinas C, Needham DM, Slooter AJC, Pandharipande PP, Watson PL, et al. Clinical Practice Guidelines for the Prevention and Management of Pain, Agitation/Sedation, Delirium, Immobility, and Sleep Disruption in Adult Patients in the ICU. Crit Care Med. [internet] 2018 Sep [acesso Mar 2018 12];46(9):825-873. Disponível em: https://www.ncbi.nlm.nih.gov/pubmed/30113379.doi: 10.1097/CCM.0000000000003299

11. Faustino TN, Pereira LC; Freitas YS; Silva RMO; Amaral JB. Prevenção e monitorização do delirium no idoso: uma intervenção educativa. Rev. Bras Enferm.[internet] 2016;69(4):678-85. Disponível em: http://www.scielo.br/scielo.php?script=sci_arttext\&pid=S003471672016000400725\&lng=pt\&tlng=pt doi: 10.1590/0034-7167.201669416

12. Bardin L. Análise de conteúdo. 3 ed. São Paulo: Edições 70, 2016, 2141p.

13. Reich R, Vieira DFVB, Lima LB, Silva ERR. Carga de trabalho em unidade coronariana segundo o Nursing Activities Score. Rev. Gaúcha Enferm. [internet] 2015; July/Sept. [acesso Dez 2018 20];36(3). 
Disponível

em:

http://www.scielo.br/scielo.php?pid=S198314472015000300028\&script=sci_arttext\&tlng=ptdoi: http://dx.doi.org/10.1590/1983-1447.2015.03.51367.

14. Luna AA, Bridi AC, Silva RCL. Delirium em terapia intensiva um estudo retrospectivo. Rev enferm UFPE. C 2015 Jan [acesso Jan 2019 20]; 9(1):69-75. doi: 10.5205/reuol.6817-60679-1ED.0901201510.

15. Frenette AJ, Bebawi ER, Deslauriers LC, Tessier AA, Perreault MM, Delisle MS, Bertrand JC, et al. Validation and comparison of CAM-ICU and ICDSC in mild and moderate traumatic brain injury patients Intensive Care Med. Epub.[internet] 2016 Jan [acesso Out 2018 20];42(1):122-3. Disponível em: https://www.ncbi.nlm.nih.gov/pubmed/26170098doi: 10.1007/s00134-015-3964.

16. Ribeiro SCL, Nascimento ERP, Lazzari DD, Jung W, Boes AA, Bertoncello KC. Conhecimento de enfermeiros sobre delirium no paciente Crítico: discurso do sujeito coletivo. Texto \& Contexto Enfermagem. [internet] 2015 abr-june [acesso Abr 2018 26];24(2):513-520. Disponível em; http://www.indexf.com/textocontexto/2015/r24513.php doi; org/10.1590/0104-07072015001702014.

17. Souza RCS. Capacitação de enfermeiros na utilização de um instrumento de avaliação de delirium. Rev Gaúcha Enferm. [internet] 2017 [acesso Out 2018 15];38(1):644-84. Disponível em: http://www.scielo.br/scielo.php?script=sci_abstract\&pid=S1983-

14472017000100801\&lng=pt\&nrm=iso\&tlng=ptdoi:10.1590/1983-1447.2017.01.64484.

18. Herdman TH. Processo de Enfermagem: um momento para relembrar seu propósito. Revista da Rede de Enfermagem do Nordeste. [internet] 2013 [acesso fev 2019 18];14(3):458-9. Disponível em: http://www.redalyc.org/articulo.oa?id=324027991001.

19. Scott P, Mcilveney F, Mallice M. Implementation of a validated delirium assessment tool in critically ill adults. Intensive Crit Care Nurs. [internet] 2013 [acesso Dez 2018 15];29(2):96-102. Disponível em: https://www.ncbi.nlm.nih.gov/pubmed/23177554doi: 10.1016 / j.icc.2012.09.001.

20. Pereira JM, Barradas FJR; Sequeira RMC, Marques MCMP, Batista MJ, Galhardas M, Santos MS. Delírium no doente crítico: fatores de risco modificáveis pelos enfermeiros. Revista de Enfermagem Referência. 2016, IV(9), abr./mai./jun: 29-36. Disponível em: http://dx.doi.org/10.12707/RIV16006

21. Projeto UTI Visitas. Implementação e avaliação da visita familiar ampliada em UTIs brasileiras. Hospital Moinhos de Vento. [internet] 2017 [acesso jan 2019 20] Disponível em: https://www.abrhrs.org.br/sites/default/files/.../case_hospital_moinhos_de_vento.pdf.

22. Souza TL, Azzolin KO, Fernandes VR. Cuidados multiprofissionais para pacientes em delirium em terapia intensiva: revisão integrativa. Rev Gaúcha Enferm. [internet] 2018 [acesso em Out 2019];39. doi: https://doi.org/10.1590/1983-1447.2018.2017-0157 
23. COFEN Resolução n 427/2012.Conselho Federal de Enfermagem. [internet] [acesso Nov 2018 20]. Disponível em: http://www.cofen.gov.br/resoluo-cofen-n-4272012_9146.html.

\section{Autor correspondente}

Katherine Pithon Oliveira

E-mail: kathypithon@hotmail.com

Endereço: Rua Jaime Brito, no 44. Centro, Itapetinga, Bahia.

CEP: $45.700-00$

\section{Contribuições de Autoria}

\section{1 - Katherine Pithon Oliveira}

Concepção do projeto, obtenção, análise e interpretação dos dados.

\section{2 - Carina Marinho Picanço}

Planejamento do projeto, acompanhamento e orientação da pesquisa, redação e análise crítica

\section{3 - Adriana Ribeiro Oliveira}

Análise, interpretação dos dados, redação e revisão crítica.

\section{4 - Ylara Idalina Silva de Assis}

Redação e análise crítica.

\section{5 - Ana Cláudia Fonseca de Souza}

Redação e análise crítica.

\section{6 - Aldacy Gonçalves Ribeiro}

Redação e análise crítica.

\section{Como citar este artigo}

Oliveira KP, Picanço CM, Oliveira AR, Assis YIS, Souza ACF, Ribeiro AG. Estratégias utilizadas por enfermeiras para minimizar a ocorrência de delirium em pacientes críticos. Rev. Enferm. UFSM. 2020 [Acesso em: Anos Mês Dia]; vol.10 e21: 1-18. DOI:https://doi.org/10.5902/2179769238788 